\title{
Minimal Social Weight User Interactions for Wearable Computers in Business Suits
}

\author{
Aaron Toney \\ Circus Systems \\ Seattle, WA \\ joeboy@hhhh.org
}

\author{
Barrie Mulley, Bruce H. Thomas, and Wayne Piekarski \\ Wearable Computer Laboratory \\ School of Computer and Information Science \\ University of South Australia \\ \{mulley,thomas,wayne\}@cs.unisa.edu.au
}

\begin{abstract}
This paper presents the e-SUIT, a wearable computer incorporated in a traditional business suit. A key feature of the system is an array of input/output devices integrated into the garment. These devices are connected to a network bus incorporated into the suit jacket. The network connects the jackets sensors and I/O with a Compaq iPAQ ${ }^{T M}$ operating Windows CE. The iPAQ is connected to the users personal information systems via a wireless LAN. Demonstrated within is an application allowing the e-SUIT to control the factory installed Microsoft Pocket Outlook ${ }^{\circledR}$. Pocket Outlook is a functioning business class application that allows the proof of concept testing to be performed on an integrated product used in the marketplace
\end{abstract}

\section{Introduction}

This paper presents the e-SUIT, a wearable computer incorporated into a traditional business suit. Figure 1 shows a user covertly entering a change to an electronic calendar appointment. This wearable computer is hidden from view, as it is embedded into the suit itself. The main goal of our system is to allow a user to strike a balance between the amount of functionality of a given technology and the social weight of that technology. We define the social weight of an item of technology to be the measure of the degradation of social interaction that occurs between the user and other people caused by the use of that item of technology. To make a wearable computing system useful within the context of the business community, these systems must interface with commercial grade business information systems. In particular we believe interacting within commercial personal information managers (PIMs), such as Microsoft Outlook, is critical to making these systems useful to the business community. The user interaction technology we have developed allows users to interact with their own Windows Outlook information system in a minimal social weight fashion. These technologies are not limited to this application, but we wish to demonstrate that these technologies are compatible with existing commercial software systems.

In a business setting, people often interact with other people in many different locations during a business day. We are interested in situations where the user is away from their office or workspace. It is in these situations that mobile computing and communication devices are most useful, and the user generally finds themselves with the least accessible infrastructure.

This section goes on to describe our assertions for a business suit. This is followed by a description of notification based systems and current known problems interacting with them. The section finishes with an explanation of the significance of social weight. We then describe our new user interface controls for the e-SUIT, and continue with a description of the implementation details for the e-SUIT. A discussion follows that focuses on what we feel are the successes of the e-SUIT and the areas that require further work and investigation. We finish the paper with some concluding remarks.

\subsection{Business suits and other uniforms}

We believe new wearable technologies to be incorporated into business wear will have to be designed to fit the uniform of the business suit. Men's business suits, which include a jacket, shirt, tie, trousers, and sometimes a vest, have been worn for almost two centuries, and they have been of a consistent design. The modern suit originated as early as the 1800 s due to the interest in classical form and the invention

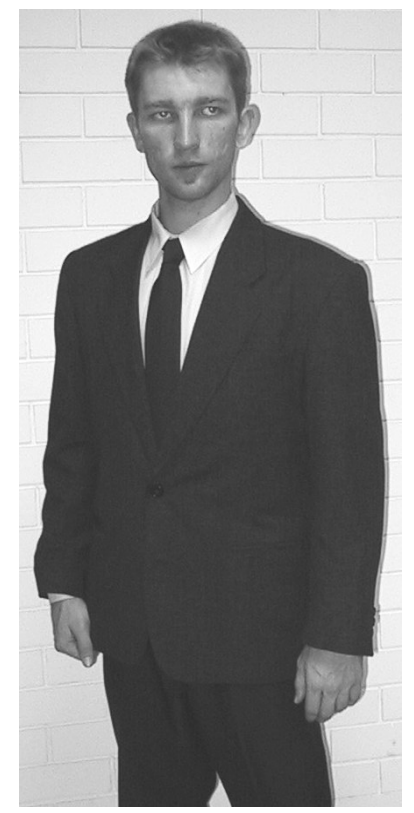

Figure 1. The e-SUIT with concealed wearable computer

In ISWC2002, 6th International Symposium on Wearable Computers

October 7-10, 2002 - Seattle, Wa, USA - Copyright (C) 2002 IEEE

Please visit http://wearables.unisa.edu.au for more information 
of the tape measure. The invention of the tape measure in 1820 allowed tailors to mass-produce ready-to-wear suits to fit anybody, which required only minor alterations. They achieved this by following the classical notion of proportion and using a tape measure. Around about 1900, the suit became a uniform for Western leaders and businessmen [1].

In the past, wearable computers have been incorporated into a number different workplace attires, such as battlefield [2], maintenance [3], and field observation [4]. In this paper we present the wearable computer incorporated into a business suit. The wearable computers for battlefield, maintenance, and field observation were visibly integrated into the apparel, and functionality of the computing systems was promoted at the expense of fashion. Sheridan et al. [5] reported the public perception of cyborgs (user's of unconcealed wearable computers) is tentative at best and, more often then not, overtly negative.

In the case of a business suit, fashion plays a much more important role. To this end, we propose that a wearable computer, which is part of the suit, should be invisible to other people unless the user chooses to use the more overt aspects of the computer. Firstly this removes the need for us to design a fashionable looking wearable computer (we leave this to the fashion designers) and secondly a piece of technology that is not noticeable to other people has an inherent low social weight. As a case in point, mobile phones have gotten smaller over the years. While the phones have been increasing in functionality, users have been taking phones off their belts and placing them into their pockets. In the case of phones, it would seem people are not changing the way they look to fit their technology.

\subsection{Interruptions, disruptions and notifications}

There is a range of applications that require some form of notification; some examples are as follows: alarms, reminders, e-mail, instant messaging, and calendars. Reminders are a form of notification and have the following two characteristic features: a signal to indicate something is to be remembered and a description to explain what needs to be remembered [6]. Current reminder systems, acting as a form of externalised memory, do not present appropriate signals at all times. More specifically, these tools are not sufficient because they are not proactive and do not make use of rich contextual information to trigger reminders at appropriate times in appropriate locations. Herstad et al. [7] claim that in order to build useful, functional and powerful tools for supporting human-human interaction, we must take context into account. CybreMinder [6] is a desktop system that determines what is the most appropriate delivery mechanism for each reminder recipient. The CybreMinder authors list a set of the features an ideal reminder tool should support: the use of rich context for specifying reminders; the ability for users and third parties to submit reminders; the ability to create reminders using a variety of input devices; the ability to receive reminders using a variety of devices; the use of reminders that include both a signal and a full description; and allowing users to view a list of all active reminders.

Notifications inherently disrupt a user's current task and social interactions. Recent research has shown notifications from instant messaging to have a generally disruptive effect during fast, stimulus-driven search tasks [8]. As an example, a user is in a meeting with a group of people and their mobile phone rings. The noise of the phone ringing produces a large social weight for the phone; the group will stop talking until the user interacts in some fashion with their phone. Vibrating motors have been introduced to provide a more covert means of informing the user of a phone call or message. The e-SUIT incorporates an extension of this idea to include a larger range of input and output devices to provide a range of interactions, from more covert to more overt, for a user with their computing environment.

Our goal is to provide users with access to a specialised range of functionality whilst maintaining the covert nature of the devices. If the user chooses to apply more functionality than the covert devices are capable of, the user then relies on their more standard computing devices, such as PDAs and notebook/desktop computers. A significant feature of this design is that the user has control of when the more overt devices are brought into play. The e-SUIT places the responsibility of appropriately adapting to the current social situation upon it's wearer.

Herstad et al. [7] field studied three different communication applications in highly mobile and communication intensive activities. Based on their studies they found the following two problems: 1) There is a need for user control of selection in the communication media during mobile communication, and 2) There is a need for user control of interaction modality when interacting with the communication application. O'Conaill and Frohlich [9] found a level of filtering of interruptions would be desirable to determine if the current interruption warranted disruption of the prior activity. This filtering can be performed with good effect in conjunction with a receptionist or secretary, i.e. a human personal assistant.

\subsection{Social Weight}

As previously mentioned, we define the social weight of an item of technology to be the measure of the degradation of social interaction that occurs between the user and other people caused by the use of that item of technology. We believe there are many factors that will increase the social weight (SW) of an item of technology, and the three factors we are focusing on in this paper are cognitive load (CL), physical presence of the device (PP), and technology apprehension (TA). We can state this as follows: $\mathrm{SW}=\mathrm{CL}+$ $\mathrm{PP}+\mathrm{TA}$. We do not claim this to be a complete measure of social weight, but it provides a useful scale when defining how much an item of technology interferes with the interaction between people. 
The goal of the e-SUIT is to minimise the social weight of associated technology for a subset of information tasks while concurrently interacting with other people. If a person chooses to get progressively more involved with an item of technology, the social weight has distinct strata within which they can move. The natural end point of this progression is the use of a PDA or Laptop, which has a relatively high social weight. This level of SW could potentially even terminate the interaction in favour of interacting with the technology.

User control over the degree of externalisation of notification signals is a significant means for allowing users graceful progression to utilize of devices with higher social weight. The user is able to use standard social protocols to determine the optimal point to use the next level of device. An example of this social protocol is that a person must excuse himself from a group, or merely say "excuse me" to a single individual, before commencing to interact with their technology. Looking again at the scenario where a mobile phone vibrates during a group meeting, the user is covertly alerted to a call or message. The predicament is that the user does have not sufficient information to determine if this transaction is worth the social weight of a pause or total break in the social interaction, both of which have a very high social weight.

We believe the SW of an item of technology can be indirectly measured. While we will not be strictly calculating the SW value for a device, we believe we can make a best estimate to group them according to value and thereby produce an approximate rank order. We present a number of indirect measures we based our estimations on. The first of these is cognitive load which may be modelled using the Model Human Processor as described in [10]. We are using loss of eye contact measured in time as an indirect measure of cognitive load. The physical presence of the device is measured by the relative size of the device. We are not measuring technology apprehension, but we believe this can be minimised by making the technology invisible to other parties in the interaction.

\section{Using the e-SUIT}

Our target application is a calendar program to facilitate communication between a user and their human personal assistant [11]. The device itself is a commercially available

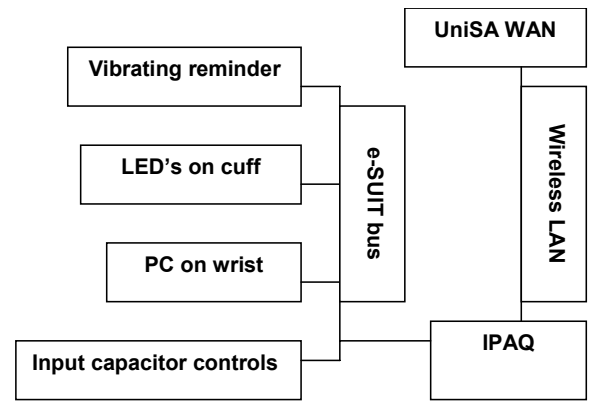

Figure 2. Basic configuration of the components
Compaq H3660 series iPAQ running Microsoft's Pocket Outlook software. The iPAQ operates on Microsoft Pocket PC 2000, a Windows CE 3.0 variant. A key assumption in the use of this application is the trust the user has with the priorities given in the calendar notifications presented to the user. In our application context, the human personal assistant is one of the few people who are able to place a priority of high on an appointment for the user. Few people would have the authority to make such an appointment, and as such the user trusts the priority given to an appointment.

\subsection{Scenario using e-SUIT}

A user is in a meeting; a vibration alarm in the shoulder of the user's jacket goes off indicating an appointment or incoming message to the user. The user then surreptitiously views a set of light emitting diodes (LEDs) in the cuff of their suit jacket to determine the priority of the information. (Red is high, yellow is medium, and green is low) Determining it is a high priority piece of information, they casually read a short message off their watch informing them of an important appointment at 2:00pm that afternoon. The user decides to accept the appointment, but needs to move the appointment two hours forward from the original starting time. The user controls the application by manipulating the capacitor slider controls on the inside of the hem of their suit jacket. The user slides his fingers along the slider until the eSUIT informs him the appointment has been moved two hours into the future. This change in starting time is signalled to the user via a coded pulsing signal from the vibration alarm device.

The user may decide to increase the SW of the interaction by reading their PDA to give them a more complete description of the appointment. They then may move onto using more intrusive technology, such as a notebook computer or desktop workstation.

The e-SUIT provides an awareness continuum along a spectrum from one extreme of peripheral awareness information (PAI) to the other of focal awareness information (FAI) [12]. Peripheral awareness information conveys information without requiring the user to take their attention away from other people in a group setting. The concept is to reduce the cognitive effort of the user. Examples of these are visual or sound cues providing information via the user's peripheral focus of attention. Focal awareness information is presented which the user can immediately respond to. Reading an appointment from a PDA or Notebook computer is a form of focal awareness information.

\subsection{Output Display Devices}

When users are sitting at a meeting table or standing in a public area, the visible features of their own bodies are their forearms, wrists, and hands, see Figure 3. We have located two visual display devices on these portions of the user's body to reduce head movement when viewing the displays, 


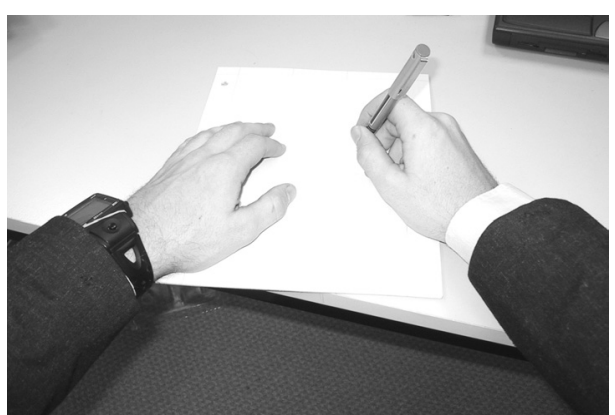

Figure 3. View of user's body while sitting at a table

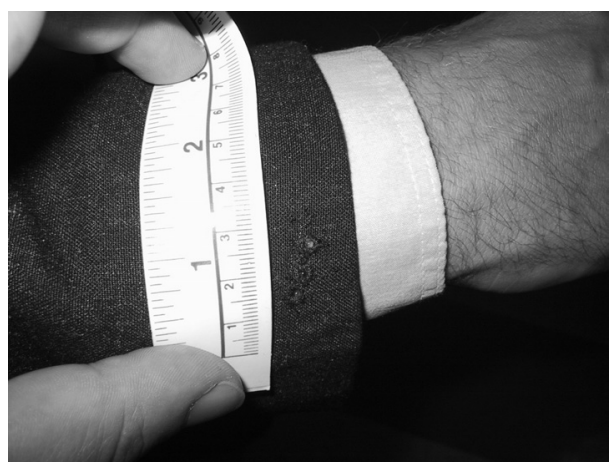

Figure 4. LED's on the e-SUIT cuff

thus reducing the devices' social weight. Coloured LEDs are sewn into the dominant handed cuff of the e-SUIT jacket, see Figure 4. These are placed so as to be visible to user when viewed straight on. The second visual display device is an LCD programmable watch to display simple text messages. A pager motor is inserted into the shoulder of the e-SUIT to provide a tactile display device. The iPAQ PDA provides the sound display, and the user has the option of removing the iPAQ PDA from their pocket to use a higher resolution display.

\section{Embedded coloured LEDs.}

Three coloured LEDs, green, yellow, and red, are placed on the jacket's cuff on the dominant hand side. We have investigated two coding strategies for theses LEDs. The first is to indicate how much time has elapsed since the user was notified of a message requiring attention. The green LED is lit first, followed by the yellow if there has been no response within two minutes, and finally the red LED is lit if there was no response after five minutes. These time values are nominally set, and further investigation is required to determine optimal values. A second strategy implements the following colour coding of the priorities for the notifications: red is high, yellow is medium, and green is low. These are just two possibilities for colour codings of the LEDs.

\section{LCD programmable watch}

We are using the onHand watch computer by Matsucom [13] to supply the display device incorporated into a watch.

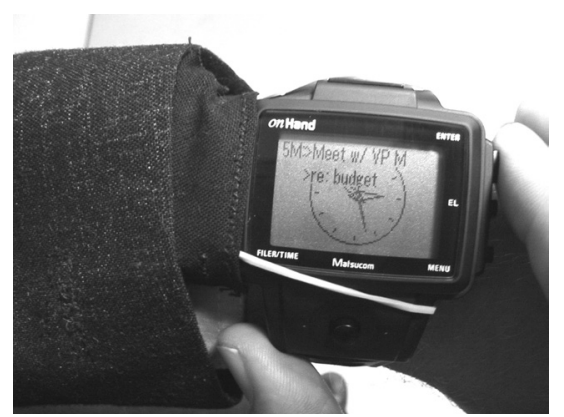

Figure 5. Watch display

The onHand's display is a 102 by 64 dot-matrix LCD with EL backlight, supporting five lines of 16 characters each. The notification application we have developed for the watch provides normal timekeeping function, and two lines of 16 characters are overlayed on the top portion of the display, see Figure 5. The IBM watch computer [14] is an excellent example of the direction this form of interaction technology is heading. Embedding new technology into existing fashionable artefacts worn currently by people. These forms of wearable computing devices have a natural low SW.

\section{Tactile display}

A pager motor is sewn into the shoulder portion of the suit jacket. This is to maintain pressure between the vibrating motor and the user's body. This display provides a simple one bit serial display for the user. We have coded seven simple signals to the user. One signal is a continuous vibration as a notification of a new message. The next six coded signals are repeating patterns to indicate the user's specified change to the length of time to move an appointment forward or the length of snooze. Table 1 specifies the different tactile patterns coded to the different time values; the time values are in seconds and symbol $\uparrow$ indicates the motor is on and $\downarrow$ indicates the motor is off. The type and number of patterns is an area of further investigation. We are not claiming these patterns are the optimal, but we do claim this form of display can covertly display information more complex than a simple "A message has arrived." Gemperle et al. proposed a similar idea, with a tactile display incorporated into an earpiece [15].

\section{Social weight of the displays}

Table 2 quantifies what we believe to be the SW ranges for the six different display devices for our system. We measure

\begin{tabular}{|l|l|}
\hline 5 mins & $0.5 \mathrm{~s} \uparrow, 1 \mathrm{~s} \downarrow$ \\
\hline 15 mins & $0.5 \mathrm{~s} \uparrow, 0.5 \mathrm{~s} \downarrow, 0.5 \mathrm{~s} \uparrow, 1 \mathrm{~s} \downarrow$ \\
\hline 30 mins & $0.5 \mathrm{~s} \uparrow, 0.5 \mathrm{~s} \downarrow, 0.5 \mathrm{~s} \uparrow, 0.5 \mathrm{~s} \downarrow, 0.5 \mathrm{~s} \uparrow, 1 \mathrm{~s} \downarrow$ \\
\hline 1 hour & $1 \mathrm{~s} \uparrow, 1.5 \mathrm{~s} \downarrow$ \\
\hline 2 hours & $1 \mathrm{~s} \uparrow, 0.5 \mathrm{~s} \downarrow, 1 \mathrm{~s} \uparrow, 1.5 \mathrm{~s} \downarrow$ \\
\hline Next day & $1 \mathrm{~s} \uparrow, 0.5 \mathrm{~s} \downarrow, 1 \mathrm{~s} \uparrow, 0.5 \mathrm{~s} \downarrow, 1 \mathrm{~s} \uparrow, 1.5 \mathrm{~s} \downarrow$ \\
\hline
\end{tabular}

Table 1. Tactile display patterns 


\begin{tabular}{|l|l|l|l|}
\cline { 2 - 4 } \multicolumn{1}{c|}{} & MHP & LOEC & PSODBU \\
\hline pagermotor & small/medium & none & none \\
\hline sound & Small & none & very large \\
\hline LEDs & Small & small & non-visible \\
\hline wrist PC & Medium & medium & small \\
\hline PDA & Large & large & medium \\
\hline laptop & very large & very large & very large \\
\hline
\end{tabular}

Table 2. Cognitive load factors for the display devices

"model human processor" (MHP) and "loss of eye contact" (LOEC) with time measurements. The following time ranges in seconds quantify the groupings as follows: none $=0 ; 0<$ small $<2 ; 2<$ medium $<5 ; 5<$ large $<15 ; 15<$ very large. As previously mentioned, these values are estimations on our part to provide a means of grouping the SW values of different devices. We based these assumptions on a reading rate of 4.35 words per second (five letters per word). We are assuming a base reaction time to be 0.38 seconds; this is the time to perform the task of seeing a visual stimulus, access an information chunk from Long-Term Memory to make decision about the stimulus, and respond by pushing a YES or NO physical button. These values can be found in Card et al. [10].

Our values in Table 2 are based on reacting to a notification signal from a calendar appointment. The pager motor has a small ( 0.5 seconds) value for the notification of a new message, and a medium (4.0 seconds) value for decoding the longest of the six different signals. The physical size of the device between users (PSODBU) is based on what we believe to be inconspicuously sized devices. We have one exception to the use of PSODBU. The PSODBU for sound is defined as the impact on people other than the user in a group situation. We place a very large value for PSODBU in the case of sound due to its large disruptive nature in the social situation.

\subsection{Input devices}

Other researchers, such as those who built the Musical Jacket [16], have investigated the placement of input control devices on and into clothing, but to our knowledge this is the first attempt to place controls into a traditional business suit. Our system provides four different sets of controls for the calendar application. These are also ranked from the highest PAI to the highest FAI as follows: 1) Capacitor buttons and sliders on the clothing; various locations are presented in this paper. 2) The buttons in the PC watch are a form of input, but we envision limited use of these in the final system. 3) Once the user has decided to break close social contact with other people, the PDA interface may be used. 4) The user may decide to use the devices with the largest SW by interacting with their notebook/desktop interface.

\section{The e-SUIT buttons.}

The e-Suit buttons are capacitive touch sensors. Fine metal thread suitable for use in tailoring was embroidered into the garment to form the buttons, see Figure 6 . Though in the future the embroidered patterns could easily be replaced with conductive fabric. When touched the capacitive value of the embroidered pad increases: potentially by several orders of magnitude. This change in capacitance can be used for the pads to measure proximity, contact, touch pressure, gross finger motion and gesture [1618]. The e-Suit currently uses the embroidered pads to implement control buttons and slider functionality. A combination of software and simple hardware filtering is then performed to filter out spurious environmental triggers.

There are two modes of slider interaction possible with the e-SUITS capacitive buttons. The first is to directly use the analog measurements of capacitance of each of the pads to extract finger geometry. The second and less complicated method is to merely treat each pad as a digital button; either pressed or not. Looking at what combination of buttons that are pressed within a window of time determining discrete slider direction and velocity. The initial implementation of the e-SUIT keyboard, described within this paper, uses the simpler later method.

We have placed the buttons on the inside of the hem of the jacket on dominant hand side of the jacket, see Figure 6 . We believe this is a good choice of position for when the user is sitting [19]. We propose a second position of inside the cuff on the non-dominant hand side (same general area as the watch) for when the user is standing. The impression we wish the user to give is they are straightening their cuff, but in reality they are controlling their computer. Areas for the placement of the buttons we dismissed are as follows: 1) inside suit pockets - they are hard to reach and uncomfortable to use, 2) inside pants pockets - they are also hard to use and socially unacceptable, and 3) on the thigh of the pants leg - we are unable to weave the controls into fabric in a fashionable way. The optimal positioning is a key research question that will require further investigation.

\section{Social weight of input devices}

This can be measured in terms of human processor time, that is to say cognitive load. Our premise is the user interface components that are closer to the PAI side of the awareness continuum, are the preferred controls, due to their lower SW. Table 3 quantifies the cognitive load factors for the four different input devices.

\section{Implementation}

The driving force behind all the technology implementations on the e-SUIT were constrained by four factors: available technology, mass production cost, care, and negligible profile to the user's daily live when not using

\begin{tabular}{|l|l|l|l|}
\cline { 2 - 4 } \multicolumn{1}{c|}{} & MHP & LOEC & PSODBU \\
\hline suit button & small/medium & None & non-visible \\
\hline wrist PC & medium & Medium & small \\
\hline PDA & large & Large & medium \\
\hline notebook & very large & very large & very large \\
\hline
\end{tabular}

Table 3. Cognitive load factors for the input devices 


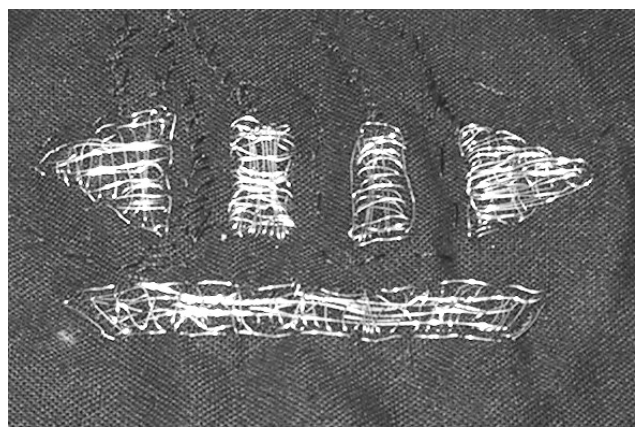

Figure 6. The e-SUIT buttons

the system. We limited ourselves to the technologies and materials that were available to bring products out onto the market today. Next we constrained on the cost of the technology. Any system that needed to be embedded in garments needs to add a negligible amount to the cost of the garment. To keep the cost down we designed using only common components and targeting a minimal component count. We estimate mass-produced in quantity of $10,000+$, the suits electronics could have a price tag as low as $\$ 17-\$ 20$ per garment (excluding IPAQ and onHand watch PC). The third constraint was that the embedded portion of the technology be maintainable with the same care that an uninstrumented garment would require. Amongst other things this would mean that our designs would need to be washable.

\subsection{Capacitive Sensors}

We base our e-SUIT buttons on capacitive sensors, and briefly this is how they operate. As charge moves through a conductor, the button in our case, in the instant after one end of a conductor is moved from its previous DC steady state voltage to a new different voltage. That moving charge has a corresponding electrostatic attraction to the objects in the immediate environment. The charge on the wire is literally electrostatically coupled to the universe. Fortunately however the strength of that coupling falls off proportionate to the cube of the distance between the coupled objects. After several tens of meters it is indistinguishable from Brownian noise. At closer distances of 0-6 centimetres however the attraction is still quite strong. It is in this region where most capacitive sensors, including those within the eSUIT function.

\section{The e-SUIT's capacitive sensors}

The e-SUIT buttons are capacitive touch sensors, which use the classic Schmidt trigger with RC feedback configuration in order to make a gyration oscillator [17]. The oscillator charges and discharges the capacitor formed by the pad touched between its power rails. This analog input is then debounced and digitised by the Schmidt trigger and fed into a frequency counter. Since a larger capacitor means a bigger "tank" to charge up, the frequency of the oscillator varies inversely proportionately to the capacitance. The eSUIT then uses a Ubicom SX18 micro controller [20] to perform the following tasks: excite the button pads, implement the frequency counters, and provide software filtering of the data before pushing out onto the e-SUIT bus.

\section{The e-SUIT's buttons}

The e-SUIT buttons are located inside the suit jacket opposite the hip, and just below the dominant hand. As the buttons are located near a relaxed resting place for the hands this placement lets the business user typically interact with the suit with a minimum of extra motion and thus has a reasonably low social weight. A button layout paralleling the hem of the jacket was chosen for the initial e-SUIT systems, as it appeared to be the layout that would cause the least social interruption through its use. The layout of the buttons is shown in Figure 6 and its placement within the jacket can be seen in Figure 12.

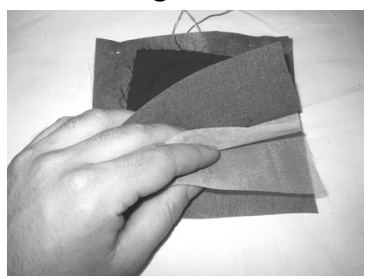

Figure 7. Layers of cloth

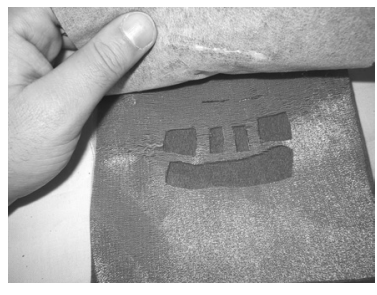

Figure 8. Button outline
The buttons themselves, as can be seen from Figure 7, are constructed in four layers. Two of the layers have electrical significance for our purposes. The first is the top layer that forms our button pads. The second is a layer of metal organza. The organza sits separated from our button pads by a layer of interfacing. Cut into the metallic organza is a pattern outlining the buttons, as shown in Figure 8. The organza helps to terminate field lines between buttons and is used to help minimize button AC coupling.

\section{So why use capacitive sensors?}

Capacitive sensors meet all of the e-SUIT's functionality requirements and our four self imposed restraints. Capacitive sensors, in particular micro controller controlled tank oscillation frequency counters like the e-SUIT uses, have both a very low component count and use components that are low cost and widely available. Further investigation is required for the e-SUIT buttons to withstand the caustic temperature and chemical process in either machine or dry cleaning. Properly sealing the micro controllers is one solution, but a substitute tactic is to be able to remove the electronics, while keeping the woven metal threads in the garment.

\subsection{Distribution of power and data}

In order to distribute power and data through out the jacket we oversewed, or bedded, fine wires in the fabric which we could then either sew into the existing garment or 


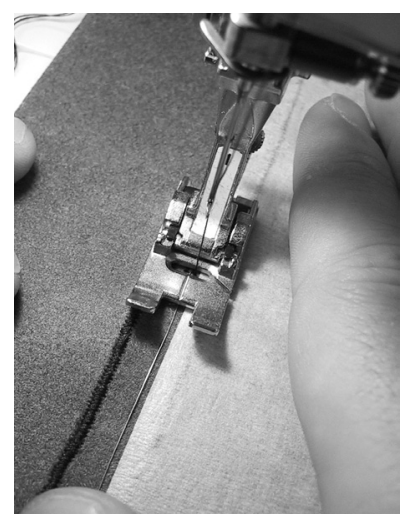

Figure 9. Run making

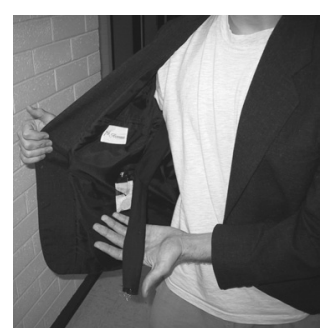

Figure 10. In the jacket

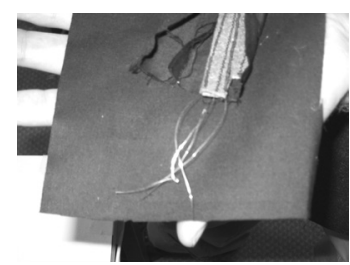

Figure 11. Closeup

use in the creation of new patches to amend the garment with. The process of bedding the wires is illustrated in Figure 9. In Figure 10 and Figure 11 we show the type of cabling we were using to run through the sleeves of the jacket. The bedding process was to lay the wires down on cloth as desired and then feed these through the sewing machine.

\subsection{Software}

The e-Suit software is driven by the functionality offered by current business tools. Integration of Microsoft Outlook into the system takes advantage of a commercial grade PIM package capable of managing contact, task and appointment data across multiple, synchronized devices. This synchronization allows users to consistently maintain the data in an environment that includes both a desktop PC that is used while the user is "in office", and handheld devices that are utilized when the user is in meetings or in other "out of office" scenarios.

A subset of this Microsoft Outlook functionality is considered for the e-Suit project. Remote/wireless synchronization allows a mobile device mounted in the suit to be synchronized with appointments made on a desktop PC. Appointment manipulation functions (reminder acknowledgement and snooze) are mapped to capacitive buttons that allow operation with gestures of low social weight. Combined, these tasks allow a user to interact with Outlook in several scenarios of interest.

The mobile device participates in two separate roles within the e-Suit the system. Firstly, it acts as a sensor that reacts to appointment data. Custom software interfaces with the Pocket Outlook Object Model (POOM) provided by the Outlook system, facilitating user interaction with the information. Secondly, the device acts as the system's Master Bus Controller (MBC) with communication and coordination responsibilities. It is connected to the system bus via a standard RS-232 serial port, transmitting and receiving

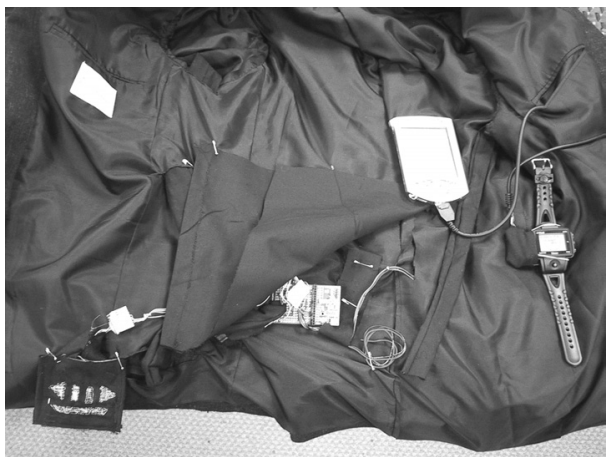

Figure 12. Inside the e-SUIT

packets from a specifically designed protocol. Microsoft eMbedded Visual $\mathrm{C}++$ tools were used in the construction of this software.

\section{Discussion}

During the construction of the current e-SUIT keyboard several methods of capacitive button detection were tried. All approaches, including the final set of buttons used with the e-SUIT experienced the plethora of problems for which capacitive circuits are famous. In fact the problems associated with capacitive detection circuits are exacerbated when used on the body; an environment, which has by its very nature, has a strong coupling to the circuit, which is in a near constant state of flux. To obtain reasonable performance both hardware to provide hysterisis on the micro controllers input and software low pass filtering were required. Currently further work is being done to add software auto calibration of the keyboard to help further reduce the effect of environmental noise.

Where else can we place items on the suit? Although the initial version of the e-SUIT was constrained to a jacket, PDA, and a watch this is by no means the limit to its domain. We predict that any successful clothing embedded wearable computer or interfaces must robustly and redundantly span multiple garments so as to not be defeated by a seasonal change in wardrobe or the simple removal of coat and hat upon entering a building. Additionally the distribution of power and data within a successful multigarment system must also be field based to eliminate the need for cabling interconnecting the garments and limiting

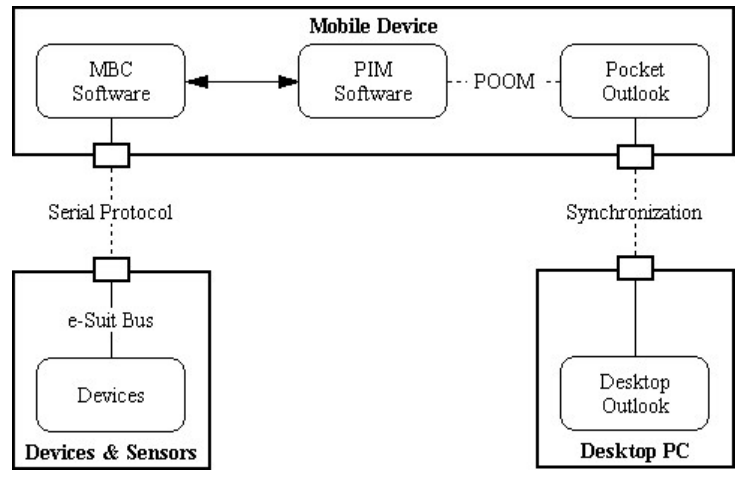

Figure 13. Overview of the software 
the users range of motion.

Where should the computing be on the e-SUIT? We have considered three options for the placement of the computing devices. Our current implementation has micro controllers embedded in the suit itself, a computer in the watch, a PDA in the suit pocket, and a wireless LAN connection to a desktop computer. The current computer on a watch can communicate with Windows Outlook. One possibility is to remove the PDA and place the functionality of the PDA onto the watch computer. Assuming the wireless LAN connection can be made to the watch computer, this would be a viable option for the user. The user would have to make a choice of on one hand not having to carry the PDA and other hand having the reduced interaction capability of the watch computer. The watch computer has a much smaller screen and more limited input technology. This solution would also constrain the user to a particular watch computer. The embedded micro controller would allow the user to pick from a number of different watch and PDA venders.

How useful is sound? Because of the high SW for sound displays that operate over public speakers, the use of the sound must only be used in the correct social circumstances. Sound could be delivered to the user via an earpiece, but that would have a SW of its own. We believe the way forward on using sound is better contextual awareness of the system. Currently the user controls when the sound is used as an alarm. If the system through the user's context could automatically determine if the SW was appropriate for sound use, this would be a large bonus to the user. Sound makes a very good ambient interface, and does not require the user to change where they visual focus.

\section{Conclusion}

We have presented the e-SUIT wearable computer that is incorporated into a traditional business suit. Wearable technologies to be incorporated into business wear will have to be designed to fit the uniform of the business suit. Men's business suits have had a consistent design for almost two centuries. Therefore these technologies must "invisible" to the public, but allow user to access their information in the mobile fashion. Key features of the e-SUIT are as follows: 1) The input and display devices have a low social weight. 2) The e-SUIT interacts with a commercial grade business information system, Microsoft's Pocket Outlook. 3) The user has control of the selection of input and output devices along an awareness continuum, from one extreme of peripheral awareness information to the other of focal awareness information.

\section{Acknowledgements}

The writers of this paper would like to acknowledge the assistance of Vina Brailsford for the lending of sewing equipment and practical tailoring advice.

\section{References}

[1] R. Dickinson, The antique uniform of business, in Christian Science Monitor, vol. 93, 2000, pp. 18.

[2] J. Murray, Wearable Computersin Battle: Recent Advances in the Land Warrior System, 4th Int'l Symposium on Wearable Computers, Atlanta, GA, 2000.

[3] D. P. Siewiorek, A. Smailagic, L. Bass, J. Siegel, and R. Martin, Adtranz: A Mobile Computing System for Maintenance and Collaboration, 2nd IEEE Int'l Conference on Wearable Computers, Pittsburgh, PA, 1998.

[4] J. Pascoe, Adding Generic Contextual Capabilities to Wearable Computers, 2nd IEEE Int'l Conference on Wearable Computers, Pittsburgh, PA, 1998.

[5] J. Sheridan, V. Lafond-Favieres, and W. Newsletter, Spectators at a Geek Show: An Ethographic Inquire into Wearable Computing, 4th Int'l Symposium on Wearable Computers, Atlanta, GA, 2000.

[6] A. K. Dey and G. D. Abowd, CybreMinder: A Context-Aware System for Supporting Reminders, 2nd Int'l Symposium on Handheld and Ubiquitous Computing, Bristol, UK, 2000.

[7] J. Herstad, D. v. Thanh, and J. Audestad, Human centered mobile communication using contextual information, Int'l Workshop on Collaboration and Mobile Computing, CMC'99, Fukushima, Japan, 1999.

[8] E. Cutrell, M. Czerwinski, and E. Horvitz, Notification, Disruption and Memory: Effects of Messaging Interruptions on Memory and Performance, HCI -Interact '01, 2001.

[9] B. O'Conaill and D. Frohlich, Timespace in the workplace: dealing with interruptions, Conference on Human Factors and Computing Systems, Denver, Colorado, 1995.

[10] S. K. Card, T. P. Moran, and A. Newell, The Psychology of Human-Computer Interaction. Hillsdale, New Jersey: Lawerence Erlbaum Associates, Inc., 1983.

[11] M. Robinson, M. Kovalainen, and E. Auramäki, Diary as dialogue in papermill process control, Communications of the ACM, vol. 43, pp. 65-70, 2000.

[12] R. S. S. Filho, Awareness and Privacy in Mobile Wearable Computers. IPADS: Interpersonal Awareness Devices, ICSInformation and Computer Science, Univ of California Irvine.

[13] Matsucom Inc., onHand User's Manual, 1st ed. 1801 Broadway, Suite 1650 Denver, Colorado 80202, 1999.

[14] C. Narayanaswami and M. T. Raghunath, Application design for a smart watch with a high resolution display, 4th Int'1 Symposium on Wearable Computers, Atlanta, GA, 2000.

[15] F. Gemperle, N. Ota, and D. Siewiorek, Design of a Wearable Tactile Display, 5th Int'1 Symp on Wearable Comp, 2001.

[16] E. R. Post, M. Orth, P. R. Russo, and N. Gershenfeld, Ebroridery: Design and fabrication of textile-based computing, IBM Systems Journal, vol. 39, pp. 840-860, 2000.

[17] L. K. Baxter, Capacitive sensors: design and applications. New York, NY: IEEE Press, 1997.

[18] J. Rekimoto, GestureWrist and GesturePad: Unobtrusive Wearable Interaction Devices, 5th Int'1 Symposium on Wearable Computers, Zurich, 2001.

[19] B. Thomas, K. Grimmer, J. Zucco, and S. Milanese, Where Does the Mouse Go? An Investigation into the Placement of a Body-Attached TouchPad Mouse for Wearable Computers, Personal and Ubiq Computing, vol. 6, pp. 113-124, 2002.

[20] Ubicom Inc, 635 Clyde Avenue, Mountain View, CA 9404322132002 . 